\title{
Ganhos genéticos para caracteres de raiz em populações de cenoura nos sistemas orgânico e convencional de produção
}

\author{
Giovani O da Silva; Jairo V Vieira; Agnaldo F de Carvalho \\ Embrapa Hortaliças, C. Postal 218,70351-970 Brasília-DF; olegario@cnph.embrapa.br; jairo@cnph.embrapa.br; agnaldo@cnph.embrapa.br
}

\begin{abstract}
RESUMO
O objetivo deste trabalho foi verificar os ganhos genéticos para caracteres de raiz em populações de cenoura cultivadas nos sistemas orgânico e convencional. Os experimentos foram conduzidos na Embrapa Hortaliças, Brasília (DF). Duas populações de cenoura derivadas da cultivar Brasília e de origem comum até 2002, foram subdivididas em duas populações, avaliadas e selecionadas, por oito gerações consecutivas, nos verões de 2000 a 2007. Em 2008, amostras de sementes das populações, provenientes de cada ciclo de seleção, foram semeadas em campo e conduzidas sob manejo orgânico e convencional de produção, em delineamento de blocos casualizados com nove tratamentos, quatro repetições e parcelas de $1 \mathrm{~m}^{2}$. Aos 90 dias após a semeadura, 20 raízes por parcela foram colhidas para a avaliação dos caracteres comprimento, diâmetro do xilema e do floema; comprimento da extensão do ombro verde; massa fresca; presença de halo; formato de ponta e de ombro e coloração do xilema e floema. Foi realizada análise de variância com determinação da interação entre tratamentos e sistemas de produção, agrupamento de médias entre os tratamentos, e calculados os ganhos reais com a seleção. Pôde-se verificar que nos oito anos de seleção não houve ganhos significativos para os caracteres estudados nas duas populações. Com isso, conclui-se que os caracteres avaliados já se encontram fixados nas duas populações estudadas. Verificou-se que a seleção não precisa ser realizada nos dois sistemas de cultivo, orgânico e convencional, possibilitando diminuição de recursos financeiros e de mão-de-obra empregados no melhoramento.
\end{abstract}

Palavras-chave: Daucus carota, interação genótipo x ambiente, progresso genético.

\begin{abstract}
Genetic gains for root characters in carrot populations in conventional and organic production systems
\end{abstract}

The objective of this work was to evaluate the genetic gain with the selection of root characters of carrot populations cultivated in organic and conventional production systems. The experiments were carried out at Embrapa Hortaliças, Brasília, Brazil. Two carrot populations derived from Brasília cultivar and with common origin until 2002, were separate in two populations and evaluated for eight generations during 2000 to 2007. In 2008, seed samples of the population in each cycle of selection were sowed in the field in both organic and conventional production systems, in a randomized blocks design with four replications of nine treatments and plots of 1 $\mathrm{m}^{2}$. After 90 days of sowing, 20 roots per plot were harvested for the evaluation of the length, xylem and phloem diameter, green shoulder length, fresh mass, presence of halo, tip and shoulder format, and the color parameters of xylem and phloem. Variance analysis was carried out to determine the interaction between treatments and production systems, grouping of means among treatments, and the real gain with the selection was estimated. In the last eight years of selection, a significant gain was not observed on the studied characters in the two populations. So we concluded that those traits are already quite developed and stabilized in both populations. The selection doesn't need to be accomplished in both areas of organic and conventional cultivation, making possible the decrease of financial and labor resources utilized in the breeding.

Keywords: Daucus carota, interaction genotype x environment, genetic progress.

(Recebido para publicação em 24 de agosto de 2011; aceito em 31 de julho de 2012) (Received on August 24, 2011; accepted on July 31, 2011)

$\mathrm{O}$ progresso genético direcionado em qualquer espécie está associado à existência de variabilidade genética, à seleção natural e/ou artificial e ao ajuste dos genótipos aos ambientes existentes. Comprovada a presença da variabilidade genética e, sobretudo o valor desta em relação à variação não-genética, a seleção assume grande importância no progresso genético. A seleção objetiva acumular alelos favoráveis a característica de interesse em determinada população e é um processo vinculado a uma constante e permanente renovação (Reis et al., 2004).

De acordo com a estratégia de seleção e o ganho proporcionado, pode-se orientar, de maneira mais efetiva, um programa de melhoramento, bem como predizer o sucesso da estratégia seletiva adotada, decidindo, com bases científicas, quais esquemas podem resultar em maior ganho genético (Cruz \& Regazzi, 2001).

Os progressos genéticos referem-se às alterações observadas nas características de interesse, durante um ciclo de seleção, com a recombinação e multiplicação das unidades selecionadas (Cruz \& Regazzi, 2001). Tais modificações ocorrerão em magnitude variada, dependendo da estratégia e dos critérios de seleção adotados. Assim, uma das atribuições mais importantes do melho- rista de plantas é identificar critérios de seleção capazes de promover alterações, no sentido desejado, nas características de interesse em um programa de melhoramento (Reis et al., 2004). Desta forma, o monitoramento do progresso obtido com a seleção se faz necessário para orientar o melhorista sobre a eficiência dos métodos de seleção empregados nas populações sob seleção.

Em decorrência do interesse dos consumidores por produtos com baixo nível de resíduos de agrotóxicos e produção menos agressiva ao meio ambiente, a agricultura agroecológica e suas derivações têm registrado um crescimento acelerado em todo o mun- 
do (Ormond et al., 2002). Segundo Lammerts Van Bueren et al. (1999), as principais diferenças que devem ser observadas no processo de melhoramento para sistemas de cultivo orgânico e convencional estão relacionadas à forma de manejo do solo, de doenças (com agentes químicos) e também às práticas de rotação de culturas. Caso esses fatores não sejam considerados durante o processo de melhoramento em sistema convencional, visando uma possível utilização das cultivares resultantes em sistema orgânico, podem proporcionar diferenças no fenótipo (desempenho) dessas cultivares. Isso porque, caso estas sejam desenvolvidas utilizando-se de práticas divergentes, dificultaria a manifestação de todo seu potencial genético sob condições de cultivo orgânico. Segundo Wolfe (2003), entre as características que apresentam desempenho inferior, quando cultivares desenvolvidas em sistema de cultivo convencional são utilizadas no sistema orgânico, estão aquelas relacionadas à nutrição da planta.

No Brasil, o melhoramento genético visando o desenvolvimento de cultivares de cenoura adaptados para cultivo em sistemas agroecológicos é incipiente. A grande maioria dos trabalhos de pesquisa, até agora realizados está direcionada para a validação de cultivares desenvolvidas para sistemas convencionais, em detrimento ao cultivo orgânico (Saminez et al., 2002; Resende et al., 2005).

A existência de diferentes sistemas de cultivo orgânico torna ainda mais complexa a tarefa dos trabalhos de melhoramento genético da cenoura para esses sistemas, sendo um processo difícil, que exige a definição de estratégias que minimizem os custos e maximizem a eficiência do processo. Algumas práticas culturais no sistema de cultivo orgânico, como a venda de cenoura em maços com as folhas ou ainda a utilização destas na produção de compostos, diminuem significativamente a quantidade de inóculo em restos culturais e a ocorrência de doenças foliares em cenoura (Souza, 2006), dificultando a seleção devido ao reduzido nível de inóculo.

Além disso, a avaliação da interação genótipos x ambientes é fundamental, pois, no caso da sua existência, há pos- sibilidades de o melhor genótipo em um ambiente não o ser em outro. Este fato influencia o ganho de seleção e dificulta a recomendação de cultivares com ampla estabilidade (Cruz \& Regazzi, 2001).

O objetivo deste trabalho foi verificar os ganhos genéticos para caracteres de raiz em populações de cenoura cultivadas nos sistemas orgânico e convencional.

\section{MATERIAL E MÉTODOS}

Duas populações de cenoura derivadas da cultivar Brasília e de origem comum até 2002, foram avaliadas e selecionadas por oito gerações consecutivas nos verões de 2000 a 2007 em sistema convencional de cultivo. A população 0712472 (população 1) foi selecionada para os seguintes caracteres: uniformidade de germinação; resistência à queima-das-folhas; reduzida porcentagem de plantas florescidas; produtividade; formato de raiz cilíndrico; ponta arredondada; comprimento de raiz entre 17 e $22 \mathrm{~cm}$, diâmetro de raiz maior que $3 \mathrm{~cm}$; cor externa laranja escuro; baixa ocorrência de halo branco no interior da raiz; baixa ocorrência de ombro verde; cor do xilema semelhante à do floema. Por sua vez, a população 0712475 (população 2) foi selecionada para uniformidade de germinação; resistência à queima-das-folhas; reduzida porcentagem de plantas florescidas; produtividade; formato de raiz cilíndrico; ponta arredondada ou semi arredondada; comprimento de raiz entre 17 e $23 \mathrm{~cm}$; diâmetro de raiz maior que $2,5 \mathrm{~cm}$; cor externa laranja ou laranja escuro; baixa ocorrência de halo branco no interior da raiz; baixa ocorrência de ombro verde; cor do xilema semelhante à do floema, e seleção para germinação a $20^{\circ} \mathrm{C}$ em laboratório. Ambas as populações foram constituídas por famílias de $1 / 2$ irmãos de polinização livre e em cada ciclo de seleção todas as raízes que apresentaram as características citadas acima foram recombinadas dando origem à geração posterior (seleção recorrente).

Parte das sementes de cada ciclo de seleção foi armazenada em câmara seca em temperatura de $6^{\circ} \mathrm{C}$ e umidade de $50 \%$, em embalagens "Polche" de alumínio. Foi realizada a determinação da germinação das sementes provenientes de cada geração em laboratório. Em 2008, amostras de sementes provenientes de cada ciclo, juntamente com a cultivar testemunha Brasília, foram semeadas no campo em dois sistemas de produção: convencional e orgânico. O delineamento utilizado foi de blocos casualizados com nove tratamentos, quatro repetições e parcelas com área total de $1 \mathrm{~m}^{2}$, a quantidade de sementes do ano de 2000 foi corrigida a 95\% de germinação, o que não foi necessário para as demais. O desbaste foi realizado 30 dias após semeadura, de modo que os espaçamentos entre plantas foi de 2 $\mathrm{cm}$ e entre linhas de $20 \mathrm{~cm}$.

Os ensaios foram conduzidos no campo experimental da Embrapa Hortaliças, em Brasília, em sistema convencional (utilizado no programa de melhoramento de cenoura) e em sistema orgânico (implantado nesse local desde 2001). A adubação no sistema convencional foi de $40 \mathrm{~kg} \mathrm{ha}^{-1}$ de nitrogênio, 12 $\mathrm{kg} \mathrm{ha}^{-1}$ de bórax e $12 \mathrm{~kg} \mathrm{ha}^{-1}$ de sulfato de zinco monohidratado, $100 \mathrm{~kg} \mathrm{ha}^{-1} \mathrm{de}$ $\mathrm{K}_{2} \mathrm{O}$ e $200 \mathrm{~kg} \mathrm{ha}^{-1}$ de $\mathrm{P}_{2} \mathrm{O}_{5}$ no plantio, e $40 \mathrm{~kg} \mathrm{ha}^{-1}$ de nitrogênio em cobertura. No sistema orgânico foi utilizado 2500 $\mathrm{kg} \mathrm{ha}^{-1}$ de composto orgânico, formulado conforme Souza \& Alcântara (2008). Não foi utilizado controle químico para doenças em ambos os sistemas. No cultivo convencional foi realizado controle de plantas daninhas em pré-emergência com Linuron (3 $\mathrm{kg} \mathrm{ha}^{-1}$ ) e Oxadiazon (3 $\mathrm{kg} \mathrm{ha}^{-1}$ ).

Aos 90 dias após a semeadura, foram colhidas 20 raízes por parcela e avaliadas individualmente para os caracteres: comprimento de raiz (cm); diâmetro da raiz, do xilema da raiz e do floema da raiz $(\mathrm{cm})$ avaliados no terço superior do comprimento da mesma; comprimento da extensão do ombro verde da raiz (cm); massa fresca da raiz (g); presença de halo (critério de notas: 1= ausência, $2=$ presença); formato de ponta da raiz (critério de notas: 1= arredondada, $2=$ levemente afilada, $3=$ afilada); formato de ombro da raiz (critério de notas: $1=$ cônico, $2=$ arredondado, $3=$ plano, $4=$ côncavo); e por leitura colorimétrica direta, determinou-se os parâmetros de coloração L* $\mathrm{a}^{*} \mathrm{~b} *$ para os tecidos xilema e floema de cada 
raiz, utilizando-se o analisador de cor de tristimulus compacto Minolta CR$200 \mathrm{~b}$ (Minolta Corporation Instrument System Division). Segundo Pereira (2002), a utilização apenas do parâmetro $a^{*}$ pode determinar o teor de $\beta$-caroteno das raízes de cenoura com segurança. $\mathrm{O}$ sistema L* a* b* Hunter é um sistema de coordenadas retangulares que define a cor em termos de luminosidade (L), vermelho versus verde (a); e amarelo versus azul (b), com esses caracteres pode-se ainda calcular o ângulo Hue (cor) e a saturação (Chroma) desta cor (Minolta, 1994).

Os dados foram submetidos à análise de homogeneidade de variância (teste de Bartlett) e de normalidade (Lilliefors). Posteriormente, foram realizadas análises de variância para cada sistema e conjunta (modelo fatorial). O agrupamento de médias entre os tratamentos foi feita por Scott-Knott (1974). Verificaram-se os ganhos genéticos reais com a seleção pela diferença de desempenho entre os diferentes tratamentos e em relação à cultivar Brasília (testemunha).

Todas as análises estatísticas foram realizadas por meio do programa Genes (Cruz, 1997).

\section{RESULTADOS E DISCUSSÃO}

Não foi possível observar norma- lidade de distribuição dos erros para os caracteres formato de ponta (FPO) e de ombro (FOM), comprimento da extensão do ombro verde (COV) e presença de halo (PHA) mesmo após transformação dos dados. Portanto, estes caracteres não foram considerados nas análises estatísticas.

Pela análise de variância conjunta (dados não mostrados), pôde-se verificar que os coeficientes de variação fenotípicos foram baixos, variando de 2,51\% para o parâmetro ' $b$ ' do xilema ( $b * X)$ até $18,19 \%$ para massa de raízes (MAS), indicando boa precisão experimental.

Para os caracteres massa de raiz (MAS), comprimento de raiz (COM), diâmetro de floema de raiz (DFR), parâmetro $\mathrm{L}^{*}$ do xilema $\left(\mathrm{L}^{*} \mathrm{X}\right)$, parâmetro $a^{*}$ do xilema $\left(a^{*} \mathrm{X}\right)$, parâmetro $\mathrm{b}^{*}$ do xilema $\left(\mathrm{b}^{*} \mathrm{X}\right)$, parâmetro $\mathrm{L}^{*}$ do floema $\left(\mathrm{L}^{*} \mathrm{~F}\right)$, parâmetro $\mathrm{a}^{*}$ do floema (a*F) e parâmetro $b^{*}$ do floema $\left(b^{*} \mathrm{~F}\right)$ houve normalidade de distribuição dos resíduos, e apenas para os caracteres diâmetro de raízes (DRA) e diâmetro do xilema da raiz (DXR) não se observaram diferenças significativas entre os ciclos de seleção; ou seja, para estes não houve progresso genético com o melhoramento. As relações entre o coeficiente de validação genético e fenotípico (CVg) $\mathrm{CV})$ também foram menores para DRA e DXR, indicando que estes caracteres são influenciados fortemente pelo ambiente, na análise feita com as duas populações conjuntamente. A informação de que DRA é um caráter influenciado fortemente pelo ambiente concorda com Silva et al. (2009b) que avaliaram famílias de cenoura em dois sistemas agroecológicos de produção. A variação de ordem genética superou a ambiental para os caracteres comprimento de raiz e caracteres de cor $a^{*}$ e b* do xilema da raiz. Vieira et al. (2009) e Silva $\&$ Vieira (2010) também verificaram variação de ordem genética influenciando o comprimento de raízes de cenoura mais fortemente do que a ambiental; enquanto que Silva \& Vieira (2010) verificaram que para os caracteres de cor a* e b* do xilema da raiz, a predominância de variabilidade genética ou ambiental variou de acordo com a população de cenoura avaliada.

$\mathrm{Na}$ avaliação da interação entre as duas populações com sistema de produção, somente o caráter DFR apresentou efeito significativo, indicando que o desempenho das populações foi semelhante nos dois sistemas de cultivo para as demais variáveis; ou seja, de modo geral, para os ambientes testados, houve consistência no comportamento dos genótipos nos sistemas convencional e orgânico. Dessa forma, a seleção pode ser feita somente em um dos sistemas,

Tabela 1. Agrupamento de médias por Scott-Knott para caracteres de raiz em oito sucessivos ciclos de seleção da População 1, em fase de melhoramento e a cultivar Brasília, cultivadas em 2008, em conjunto para sistemas convencional e orgânico de produção (means clustered by Scott-Knott for root characters, in eight successive cycles of selection in one carrot population (Population 1) in breeding phase and the cultivar Brasília (released in 1981) in wich the population was originated, cultivated in 2008, jointly to conventional and organic production systems). Brasília, Embrapa Hortaliças, 2008.

\begin{tabular}{lccccccccccc}
\hline Populações & MAS & COM & DRA & DXR & DFR & $\mathbf{L}^{*} \mathbf{X}$ & $\mathbf{A}^{*} \mathbf{X}$ & $\mathbf{B}^{*} \mathbf{X}$ & $\mathbf{L}^{*} \mathbf{F}$ & $\mathbf{A}^{*} \mathbf{F}$ & $\mathbf{B} * \mathbf{F}$ \\
\hline 'Brasília' & $78,36 \mathrm{a}$ & $13,63 \mathrm{~d}$ & $2,87 \mathrm{a}$ & $1,47 \mathrm{a}$ & $1,41 \mathrm{~b}$ & $38,18 \mathrm{a}$ & $11,24 \mathrm{c}$ & $42,55 \mathrm{c}$ & $38,54 \mathrm{a}$ & $21,35 \mathrm{a}$ & $49,45 \mathrm{~b}$ \\
$2000^{*}$ & $63,02 \mathrm{a}$ & $15,11 \mathrm{~b}$ & $2,52 \mathrm{~b}$ & $1,12 \mathrm{~b}$ & $1,41 \mathrm{~b}$ & $39,04 \mathrm{a}$ & $19,53 \mathrm{~b}$ & $48,15 \mathrm{~b}$ & $41,01 \mathrm{a}$ & $23,02 \mathrm{a}$ & $52,05 \mathrm{a}$ \\
$2001^{*}$ & $71,02 \mathrm{a}$ & $16,24 \mathrm{a}$ & $2,53 \mathrm{~b}$ & $1,10 \mathrm{~b}$ & $1,44 \mathrm{~b}$ & $38,94 \mathrm{a}$ & $20,23 \mathrm{a}$ & $48,98 \mathrm{a}$ & $40,06 \mathrm{a}$ & $22,64 \mathrm{a}$ & $51,21 \mathrm{a}$ \\
$2002^{*}$ & $67,18 \mathrm{a}$ & $15,62 \mathrm{~b}$ & $2,52 \mathrm{~b}$ & $1,12 \mathrm{~b}$ & $1,41 \mathrm{~b}$ & $38,72 \mathrm{a}$ & $20,53 \mathrm{a}$ & $48,79 \mathrm{a}$ & $40,95 \mathrm{a}$ & $23,57 \mathrm{a}$ & $52,50 \mathrm{a}$ \\
2003 & $67,69 \mathrm{a}$ & $14,48 \mathrm{c}$ & $2,68 \mathrm{a}$ & $1,15 \mathrm{~b}$ & $1,53 \mathrm{a}$ & $39,21 \mathrm{a}$ & $19,50 \mathrm{~b}$ & $48,15 \mathrm{~b}$ & $40,45 \mathrm{a}$ & $22,89 \mathrm{a}$ & $51,63 \mathrm{a}$ \\
2004 & $68,91 \mathrm{a}$ & $15,35 \mathrm{~b}$ & $2,45 \mathrm{~b}$ & $0,98 \mathrm{~b}$ & $1,47 \mathrm{a}$ & $38,34 \mathrm{a}$ & $20,42 \mathrm{a}$ & $48,18 \mathrm{~b}$ & $40,95 \mathrm{a}$ & $23,51 \mathrm{a}$ & $52,20 \mathrm{a}$ \\
2005 & $72,87 \mathrm{a}$ & $15,20 \mathrm{~b}$ & $2,65 \mathrm{a}$ & $1,16 \mathrm{~b}$ & $1,50 \mathrm{a}$ & $38,66 \mathrm{a}$ & $20,25 \mathrm{a}$ & $48,97 \mathrm{a}$ & $40,29 \mathrm{a}$ & $22,88 \mathrm{a}$ & $52,19 \mathrm{a}$ \\
2006 & $73,73 \mathrm{a}$ & $15,93 \mathrm{a}$ & $2,64 \mathrm{a}$ & $1,13 \mathrm{~b}$ & $1,52 \mathrm{a}$ & $38,89 \mathrm{a}$ & $21,02 \mathrm{a}$ & $49,11 \mathrm{a}$ & $41,52 \mathrm{a}$ & $23,81 \mathrm{a}$ & $52,32 \mathrm{a}$ \\
2007 & $69,51 \mathrm{a}$ & $14,85 \mathrm{c}$ & $2,45 \mathrm{~b}$ & $1,02 \mathrm{~b}$ & $1,44 \mathrm{~b}$ & $38,71 \mathrm{a}$ & $20,76 \mathrm{a}$ & $49,20 \mathrm{a}$ & $39,91 \mathrm{a}$ & $22,80 \mathrm{a}$ & $51,61 \mathrm{a}$ \\
\hline
\end{tabular}

$\mathrm{MAS}=$ massa; $\mathrm{COM}=$ comprimento; $\mathrm{DRA}=$ diâmetro da raiz; $\mathrm{DXR}=$ diâmetro do xilema; $\mathrm{DFR}=$ diâmetro do floema; $\mathrm{L} * \mathrm{X}=$ parâmetro $\mathrm{L} *$ do xilema; $A^{*} X=$ parâmetro $A^{*}$ do xilema; $B * X=$ parâmetro $B^{*}$ do xilema; $L^{*} F=$ parâmetro $L^{*}$ do floema; $A * F=$ parâmetro $A *$ do floema; $\mathrm{B} * \mathrm{~F}=$ parâmetro $\mathrm{B}^{*}$ do floema $\left(\mathrm{MAS}=\right.$ mass; $\mathrm{COM}=$ lenght; $\mathrm{DRA}=$ root diameter; $\mathrm{DXR}=$ xylem diameter; $\mathrm{DFR}=\mathrm{phloem}$ diameter; $\mathrm{L}^{*} \mathrm{X}=$ xylem $L^{*}$ parameter; $A * X=$ xylem $A^{*}$ parameter; $B * X=$ xylem $B^{*}$ parameter; $L^{*} F=$ phloem $L^{*}$ parameter; $A^{*} F=$ phloem $A *$ parameter; $\mathrm{B} * \mathrm{~F}=$ phloem $\mathrm{B} *$ parameter); * gerações em comum para as duas populações que foram separadas em 2002 (*generations in common for the two populations that were separated in 2002). 
Tabela 2. Agrupamento de médias por Scott-Knott para caracteres de raiz em oito sucessivos ciclos de seleção da População 2, em fase de melhoramento e a cultivar Brasília, cultivadas em 2008, em conjunto para sistemas convencional e orgânico de produção (means clustered by Scott-Knott to root characters, in eight successive cycles of selection to one carrot population (Population 2) in breeding phase and the cultivar Brasília (released in 1981) in which the population was originated, cultivated in 2008, jointly to conventional and organic production systems). Brasília, Embrapa Hortaliças, 2008.

\begin{tabular}{lccccccccccc}
\hline Populaçóes & MAS & COM & DRA & DXR & DFR & $\mathbf{L} * \mathbf{X}$ & $\mathbf{A} * \mathbf{X}$ & $\mathbf{B} * \mathbf{X}$ & $\mathbf{L} * \mathbf{F}$ & $\mathbf{A}^{*} \mathbf{F}$ & $\mathbf{B}^{*} \mathbf{F}$ \\
\hline 'Brasília' & $78,36 \mathrm{a}$ & $13,63 \mathrm{c}$ & $2,88 \mathrm{a}$ & $1,47 \mathrm{a}$ & $1,41 \mathrm{a}$ & $38,18 \mathrm{~b}$ & $11,24 \mathrm{~d}$ & $42,55 \mathrm{~d}$ & $38,54 \mathrm{~b}$ & $21,35 \mathrm{~b}$ & $49,45 \mathrm{~b}$ \\
$2000^{*}$ & $63,02 \mathrm{a}$ & $15,11 \mathrm{~b}$ & $2,53 \mathrm{~b}$ & $1,12 \mathrm{~b}$ & $1,41 \mathrm{a}$ & $39,04 \mathrm{a}$ & $19,53 \mathrm{~b}$ & $48,15 \mathrm{a}$ & $41,01 \mathrm{a}$ & $23,02 \mathrm{a}$ & $52,05 \mathrm{a}$ \\
$2001^{*}$ & $71,02 \mathrm{a}$ & $16,24 \mathrm{~b}$ & $2,53 \mathrm{~b}$ & $1,10 \mathrm{~b}$ & $1,44 \mathrm{a}$ & $38,94 \mathrm{a}$ & $20,23 \mathrm{a}$ & $48,98 \mathrm{a}$ & $40,06 \mathrm{a}$ & $22,64 \mathrm{a}$ & $51,21 \mathrm{a}$ \\
$2002^{*}$ & $67,18 \mathrm{a}$ & $15,62 \mathrm{~b}$ & $2,52 \mathrm{~b}$ & $1,12 \mathrm{~b}$ & $1,41 \mathrm{a}$ & $38,72 \mathrm{a}$ & $20,53 \mathrm{a}$ & $48,79 \mathrm{a}$ & $40,95 \mathrm{a}$ & $23,57 \mathrm{a}$ & $52,50 \mathrm{a}$ \\
2003 & $83,93 \mathrm{a}$ & $16,20 \mathrm{~b}$ & $2,57 \mathrm{~b}$ & $1,13 \mathrm{~b}$ & $1,44 \mathrm{a}$ & $37,53 \mathrm{~b}$ & $19,15 \mathrm{~b}$ & $47,00 \mathrm{~b}$ & $40,22 \mathrm{a}$ & $22,90 \mathrm{a}$ & $51,19 \mathrm{a}$ \\
2004 & $76,65 \mathrm{a}$ & $16,07 \mathrm{~b}$ & $2,48 \mathrm{~b}$ & $1,15 \mathrm{~b}$ & $1,33 \mathrm{a}$ & $37,18 \mathrm{~b}$ & $17,14 \mathrm{c}$ & $44,81 \mathrm{c}$ & $40,31 \mathrm{a}$ & $21,07 \mathrm{~b}$ & $49,77 \mathrm{~b}$ \\
2005 & $74,64 \mathrm{a}$ & $16,00 \mathrm{~b}$ & $2,57 \mathrm{~b}$ & $1,17 \mathrm{~b}$ & $1,39 \mathrm{a}$ & $37,80 \mathrm{~b}$ & $17,97 \mathrm{c}$ & $45,87 \mathrm{c}$ & $38,62 \mathrm{~b}$ & $20,56 \mathrm{~b}$ & $49,23 \mathrm{~b}$ \\
2006 & $80,74 \mathrm{a}$ & $17,30 \mathrm{a}$ & $2,64 \mathrm{~b}$ & $1,21 \mathrm{~b}$ & $1,43 \mathrm{a}$ & $37,57 \mathrm{~b}$ & $18,10 \mathrm{c}$ & $45,71 \mathrm{c}$ & $40,07 \mathrm{a}$ & $22,43 \mathrm{a}$ & $50,86 \mathrm{a}$ \\
2007 & $72,05 \mathrm{a}$ & $15,61 \mathrm{~b}$ & $2,39 \mathrm{~b}$ & $1,01 \mathrm{~b}$ & $1,38 \mathrm{a}$ & $38,19 \mathrm{~b}$ & $18,18 \mathrm{c}$ & $46,39 \mathrm{c}$ & $39,89 \mathrm{a}$ & $21,38 \mathrm{~b}$ & $50,12 \mathrm{~b}$ \\
\hline
\end{tabular}

$\mathrm{MAS}=$ massa; $\mathrm{COM}=$ comprimento; $\mathrm{DRA}=$ diâmetro da raiz; $\mathrm{DXR}=$ diâmetro do xilema; $\mathrm{DFR}=$ diâmetro do floema; $\mathrm{L}^{*} \mathrm{X}=$ parâmetro $\mathrm{L}^{*}$ do xilema; $A^{*} X=$ parâmetro $A^{*}$ do xilema; $B^{*} X=$ parâmetro $B^{*}$ do xilema; $L^{*} F=$ parâmetro $L^{*}$ do floema; $A^{*} F=$ parâmetro $A^{*}$ do floema; $\mathrm{B}^{*} \mathrm{~F}=$ parâmetro $\mathrm{B}^{*}$ do floema $\left(\mathrm{MAS}=\right.$ mass; $\mathrm{COM}=$ lenght; $\mathrm{DRA}=$ root diameter; $\mathrm{DXR}=$ xylem diameter; $\mathrm{DFR}=$ phloem diameter; $\mathrm{L}^{*} \mathrm{X}=$ xylem $L^{*}$ parameter; $A^{*} X=$ xylem $A^{*}$ parameter; $B^{*} X=$ xylem $B^{*}$ parameter; $L^{*} F=$ phloem $L^{*}$ parameter; $A^{*} F=$ phloem $A^{*}$ parameter; $\mathrm{B} * \mathrm{~F}=$ phloem $\mathrm{B} *$ parameter); *gerações em comum para as duas populações que foram separadas em 2002 (*generations in commom for the two populations that were separated in 2002).

Tabela 3. Porcentagem de aumento ou decréscimo das médias dos caracteres de raiz em oito sucessivos ciclos de seleção da População 1, em fase de melhoramento, e a população comercial Brasília, cultivadas em 2008, em conjunto para sistema de produção convencional e orgânico (increase or decrease percentage of means to characters in successive cycles of selection, relatively to Brasília cultivar in which the population was originated, in successive cycles of selection to one carrot population (Population 1) in breeding phase, cultivated in 2008, jointly to conventional and organic production systems). Brasília, Embrapa Hortaliças, 2008.

\begin{tabular}{lccrrrrrllll}
\hline Populações & MAS & COM & \multicolumn{1}{c}{ DRA } & \multicolumn{1}{c}{ DXR } & \multicolumn{1}{c}{ DFR } & L*X & A*X & B*X & L*F & $\mathbf{A}^{*} \mathbf{F}$ & $\mathbf{B}^{*} \mathbf{F}$ \\
\hline BSB & 100,00 & 100,00 & 100,00 & 100,00 & 100,00 & 100,00 & 100,00 & 100,00 & 100,00 & 100,00 & 100,00 \\
$2000^{*}$ & 80,42 & 110,88 & 87,90 & 76,01 & 100,27 & 102,25 & 173,80 & 113,17 & 106,42 & 107,79 & 105,25 \\
$2001^{*}$ & 90,63 & 119,16 & 87,96 & 74,69 & 101,77 & 101,98 & 180,05 & 115,11 & 103,93 & 106,03 & 103,55 \\
$2002^{*}$ & 85,73 & 114,60 & 87,73 & 76,01 & 99,91 & 101,41 & 182,71 & 114,67 & 106,24 & 110,37 & 106,15 \\
2003 & 86,38 & 106,21 & 93,31 & 78,57 & 108,64 & 102,68 & 173,52 & 113,16 & 104,94 & 107,18 & 104,41 \\
2004 & 87,95 & 112,59 & 85,21 & 66,63 & 104,54 & 100,42 & 181,70 & 113,22 & 106,25 & 110,11 & 105,55 \\
2005 & 93,00 & 111,51 & 92,29 & 78,91 & 106,21 & 101,27 & 180,19 & 115,10 & 104,53 & 107,15 & 105,54 \\
2006 & 94,09 & 116,84 & 91,96 & 77,03 & 107,49 & 101,84 & 187,08 & 115,42 & 107,73 & 111,49 & 105,80 \\
2007 & 88,71 & 108,96 & 85,49 & 69,28 & 102,35 & 101,38 & 184,79 & 115,63 & 103,56 & 106,76 & 104,36 \\
\hline
\end{tabular}

$\mathrm{MAS}=$ massa; $\mathrm{COM}=$ comprimento; $\mathrm{DRA}=$ diâmetro da raiz; $\mathrm{DXR}=$ diâmetro do xilema; $\mathrm{DFR}=$ diâmetro do floema; $\mathrm{L} * \mathrm{X}=$ parâmetro $\mathrm{L}^{*}$ do xilema; $A * X=$ parâmetro $A *$ do xilema; $B * X=$ parâmetro $B *$ do xilema; $L^{*} F=$ parâmetro $L^{*}$ do floema; $A * F=$ parâmetro $A *$ do floema; $\mathrm{B} * \mathrm{~F}=$ parâmetro $\mathrm{B}^{*}$ do floema $\left(\mathrm{MAS}=\right.$ mass; $\mathrm{COM}=$ lenght; $\mathrm{DRA}=$ root diameter; $\mathrm{DXR}=$ xylem diameter; $\mathrm{DFR}=$ phloem diameter; $\mathrm{L}^{*} \mathrm{X}=$ xylem $L^{*}$ parameter; $A^{*} X=$ xylem $A^{*}$ parameter; $B * X=$ xylem $B *$ parameter; $L^{*} F=$ phloem $L^{*}$ parameter; $A * F=$ phloem $A *$ parameter; $\mathrm{B} * \mathrm{~F}=$ phloem $\mathrm{B} *$ parameter); *gerações em comum para as duas populações que foram separadas em 2002 (*generations in commom for the two populations that were separated in 2002).

com economia de recursos financeiros e de mão-de-obra, o que não seria recomendado apenas para DFR. Além disso, a não significância da interação possibilita ganhos maiores durante o processo de melhoramento e o desenvolvimento de cultivares com ampla estabilidade (Cruz \& Regazzi, 2001). Estes resultados indicam que as afirmações de Wolfe (2003) "a seleção de genótipos ou de uma população para cultivo em sistemas orgânicos deve considerar o desempenho destes em vários ambientes", e de Lammerts Van Bueren et al. (1999) "o melhoramento genético para cultivo orgânico deve ser feito para cada local ou região, em função de sistemas de produção muito diversos", podem não ser válidas para todos os casos. Ressalta-se que estas conclusões são válidas para as populações em estudo, que tiveram origem comum a partir da cultivar Brasília.

Da mesma forma, Silva et al. (2009) avaliaram os caracteres COM, DRA, MAS, DXR, DRA relação entre DXR/ DRA, A*X e A*F, em uma população da cultivar Brasília, cultivada nos modelos de Agricultura Natural e Agricultura Orgânica, no Distrito Federal, e verificaram comportamento semelhante dos genótipos estudados concluindo que o melhoramento pode ser efetuado em 
Tabela 4. Porcentagem de aumento ou decréscimo das médias dos caracteres de raiz em oito sucessivos ciclos de seleção da População 2, em fase de melhoramento, e a população comercial Brasília, cultivadas em 2008, em conjunto para sistema de produção convencional e orgânico (increase or decrease percentage of means to characters in successive cycles of selection, relatively to Brasília cultivar in which the population was originated, in successive cycles of selection to one carrot population (Population 2) in breeding phase, cultivated in 2008, jointly to conventional and organic production systems). Brasília, Embrapa Hortaliças, 2008.

\begin{tabular}{lrcrrrrrrrrr}
\hline Populações & \multicolumn{1}{c}{ MAS } & COM & \multicolumn{1}{c}{ DRA } & \multicolumn{1}{c}{ DXR } & \multicolumn{1}{c}{ DFR } & \multicolumn{1}{c}{$\mathbf{L}^{*} \mathbf{X}$} & $\mathbf{A} * \mathbf{X}$ & $\mathbf{B} * \mathbf{X}$ & $\mathbf{L} * \mathbf{F}$ & $\mathbf{A}^{*} \mathbf{F}$ & $\mathbf{B} * \mathbf{F}$ \\
\hline BSB & 100,00 & 100,00 & 100,00 & 100,00 & 100,00 & 100,00 & 100,00 & 100,00 & 100,00 & 100,00 & 100,00 \\
$2000^{*}$ & 80,42 & 110,88 & 87,90 & 76,01 & 100,27 & 102,25 & 173,80 & 113,17 & 106,42 & 107,79 & 105,25 \\
$2001 *$ & 90,63 & 119,16 & 87,96 & 74,69 & 101,77 & 101,98 & 180,05 & 115,11 & 103,93 & 106,03 & 103,55 \\
$2002 *$ & 85,73 & 114,60 & 87,73 & 76,01 & 99,91 & 101,41 & 182,71 & 114,67 & 106,24 & 110,37 & 106,15 \\
2003 & 107,11 & 118,87 & 89,22 & 76,99 & 101,95 & 98,29 & 170,46 & 110,45 & 104,36 & 107,26 & 103,51 \\
2004 & 97,82 & 117,89 & 86,23 & 78,27 & 94,50 & 97,39 & 152,54 & 105,31 & 104,59 & 98,66 & 100,63 \\
2005 & 95,25 & 117,37 & 89,18 & 79,85 & 98,89 & 99,00 & 159,97 & 107,79 & 100,20 & 96,27 & 99,55 \\
2006 & 103,04 & 126,91 & 91,74 & 82,23 & 101,64 & 98,39 & 161,09 & 107,43 & 103,98 & 105,05 & 102,85 \\
2007 & 91,94 & 114,53 & 82,92 & 68,77 & 97,65 & 100,01 & 161,80 & 109,02 & 103,50 & 100,13 & 101,35 \\
\hline
\end{tabular}

$\mathrm{MAS}=$ massa; $\mathrm{COM}=$ comprimento; $\mathrm{DRA}=$ diâmetro da raiz; $\mathrm{DXR}=$ diâmetro do xilema; $\mathrm{DFR}=$ diâmetro do floema; $\mathrm{L}^{*} \mathrm{X}=$ parâmetro $\mathrm{L} *$ do xilema; $A^{*} X=$ parâmetro $A^{*}$ do xilema; $B * X=$ parâmetro $B *$ do xilema; $L * F=$ parâmetro $L^{*}$ do floema; $A * F=$ parâmetro $A *$ do floema; $\mathrm{B} * \mathrm{~F}=$ parâmetro $\mathrm{B} *$ do floema $(\mathrm{MAS}=$ mass; $\mathrm{COM}=$ lenght; $\mathrm{DRA}=$ root diameter; $\mathrm{DXR}=$ xylem diameter; $\mathrm{DFR}=\mathrm{phloem}$ diameter; $\mathrm{L} * \mathrm{X}=$ xylem $L^{*}$ parameter; $A^{*} X=$ xylem $A^{*}$ parameter; $B * X=$ xylem $B^{*}$ parameter; $L^{*} F=$ phloem $L^{*}$ parameter; $A^{*} F=$ phloem $A *$ parameter; $\mathrm{B}^{*} \mathrm{~F}=$ phloem $\mathrm{B} *$ parameter); *gerações em comum para as duas populações que foram separadas em 2002 (*generations in commom for the two populations that were separated in 2002).

apenas um dos sistemas.

Considerando apenas sistemas convencionais, inconsistência de comportamento de genótipos com as variações ambientais foram verificadas por Vieira \& Oliveira (2003) em nove populações de cenoura do grupo Brasília em três locais: Brasília (DF), São Gotardo (MG) e Irecê (BA), para os caracteres MAS, COM e DRA. Os autores verificaram que a presença de interação de elevada magnitude para MAS e DRA foi devida predominantemente à parte simples da interação, indicando que o desenvolvimento de cultivares para os três locais poderia ser efetuado em apenas um ambiente.

No presente estudo as interações para os parâmetros de cor $\mathrm{L}^{*} \mathrm{a}^{*} \mathrm{~b}^{*}$ discutidas acima foram calculadas entre os sistemas de cultivo orgânico e convencional, pertencentes à mesma estação experimental. Simon \& Peterson (1993) observaram que os níveis de carotenóides determinados por espectofotometria, em diferentes cultivares de cenoura foram significativamente influenciados por condições edafoclimáticas de diferentes regiões de plantio convencional nos Estados Unidos, indicando que interações significativas podem ser possíveis quando variação maior for observada em relação ao ambiente.

Como não houve interação significa- tiva entre as populações avaliadas e os sistemas de cultivo, com exceção para DFR, as comparações de médias para as duas populações de cenoura foram feitas com a média dos dois sistemas de cultivo. Tanto para a população 1 (Tabela 1) quanto para a população 2 (Tabela 2), as médias do caráter MAS foram alocadas em um mesmo grupo pelo teste de Scott-Knott a 5\%, indicando que a seleção não proporcionou ganhos significativos. Como este é um caráter quantitativo e associado ao DRA, DXR e COM (Silva et al., 2012), pode-se analisar os ganhos para estes caracteres componentes. Para ambas as populações, as médias também não foram significativamente diferentes para os caracteres DFR, $\mathrm{L}^{*} \mathrm{X}, \mathrm{L} * \mathrm{~F}$ e $\mathrm{a}^{*} \mathrm{~F}$, indicando que não foi possível obter alteração com a seleção recorrente aplicada para estes caracteres.

A metodologia normalmente utilizada na seleção para maior teor de $\beta$-caroteno é a seleção visual para raízes com coloração alaranjada mais escura, pois de acordo com Michalik et al. (1985) há associação entre a pigmentação mais intensa das raízes, especialmente na parte interna, e o maior conteúdo de caroteno, sendo esta uma metodologia mais prática e viável dada a grande quantidade de raízes que são selecionadas a cada ciclo em programas de melhoramento. No entanto, pequenas variações de tonalidade não podem ser visualizadas a olho nu. Os parâmetros de cor $\mathrm{L}^{*} \mathrm{a}^{*} \mathrm{~b}^{*}$ podem ser utilizados para calcular o ângulo Hue (cor) e a saturação (Chroma) desta cor, conforme recomendado por Minolta (1994), podendo ser indicativos do teor de $\beta$-caroteno das raízes de cenoura. Porém, a utilização apenas do parâmetro a* também pode determinar o teor deste pigmento em cenoura com certa segurança (Pereira, 2002). Característica esta que é muito valorizada pelos consumidores no momento da compra da cenoura (Onoyama et al. 2011).

O resultado mostrando a inexistência de diferença significativa para $a * F$ na população 1 e ainda pequena diferença para a população 2 , indica que a seleção visual que foi aplicada nas sucessivas gerações, foi eficiente apenas na seleção para a coloração na parte da raiz formada pelo xilema. Isso pode ter ocorrido pelo fato de que normalmente, nas populações do grupo Brasília, a coloração do tecido do floema é mais intensa que para o xilema (Silva \& Vieira, 2008; Silva \& Vieira, 2010). No presente estudo os valores médios para cada população em fase de melhoramento mais a cultivar Brasília, variaram de 11,23 a 21,02 para o a*X e de 21,35 a 23,81 para a*F.

Em relação à cultivar Brasília, para o caráter $\mathrm{a}^{*} \mathrm{X}$, nas populações 1 e 2 , os 
ganhos foram de 84,79 e $61,80 \%$, respectivamente (Tabelas 3 e 4), indicando que um grande ganho foi obtido com a seleção visual para esta porção da raiz.

Silva \& Vieira (2010), avaliaram o progresso com a seleção efetuada por seis sucessivos ciclos de seleção em três populações de cenoura e verificaram que não foi possível obter êxito na seleção visual, provavelmente devido à baixa variabilidade de ordem genética verificada, visto que as raízes das populações já estavam bastante escuras, indicando grande quantidade de $\beta$-caroteno.

Ganhos puderam ser verificados em relação a cultivar Brasília, mas não entre as oito gerações de seleção, para os caracteres DXR e b*F, indicando que desde o lançamento da cultivar Brasília em 1981, modificações significativas puderam ser obtidas para estes caracteres. Entretanto, nos últimos ciclos de seleção não houve ganhos significativos. Quanto ao DXR, verificou-se que a partir da cv. Brasília o diâmetro do xilema das raízes diminuiu, o mesmo ocorrendo para DRA na população 2. Considerando que estes caracteres são de grande importância na obtenção de raízes mais grossas e com maior rendimento, constata-se que é necessária a seleção destes caracteres em conjunto com o rendimento. O DXR da cv. Brasília até 2000 diminuiu 23,99\% enquanto que $\mathrm{b} * \mathrm{~F}$ aumentou em 5,25\% nas duas populações.

$O$ fato de MAS ter se mantido constante nas oitos gerações, mesmo com a diminuição no DXR, bem como a diminuição no DRA nas duas populações (12,1\% da cv Brasília até 2002), parece ser devido ao aumento no comprimento das raízes que foi de 8,96 e 14,53\% para as populações 1 e 2 , respectivamente. Vale ressaltar que a raiz cresce primeiramente em comprimento e só depois em diâmetro (White \& Strandberg, 1978; Vieira et al., 2012). Da mesma forma, Silva \& Vieira (2010), verificaram que a seleção para maior massa e comprimento de raiz, pode levar à diminuição do caráter diâmetro de raiz; estes autores observaram também decréscimo nos valores do diâmetro do floema da raiz. De acordo com Silva \& Vieira (2008) o rendimento de raízes é influenciado fortemente pelo diâmetro da raiz e do xilema da raiz. Sendo assim podemos concluir que estes caracteres devem ser considerados conjuntamente no momento da seleção.

Silva \& Vieira (2009) estudaram os avanços com a seleção de uma população de cenoura do grupo Brasília por seis gerações consecutivas e verificaram que houve ganhos superiores apenas nos primeiros ciclos de seleção para diminuição das raízes refugo e o aumento da resistência à queima-das-folhas. Sendo que a quantidade de raízes classificadas como comerciais aumentou durante todos os ciclos de seleção, tanto com ombro quanto sem ombro verde, o ganho foi maior para as raízes sem ombro verde.

Desta forma, pôde-se verificar que nos oito anos de seleção não houve ganho expressivo para os caracteres estudados nas duas populações. Com isso, conclui-se que os caracteres avaliados no presente trabalho já se encontram fixados nas duas populações. Quanto aos parâmetros de cor, as raízes das duas populações avaliadas já se apresentam com coloração bastante escura, com evolução muito grande desde a criação da cv. Brasília. Verifica-se ainda que o comprimento, a massa e o diâmetro de raiz, devem ser selecionados conjuntamente para evitar a obtenção de raízes muito finas. A informação de que o melhoramento não precisa ser realizado em ambas as áreas de cultivo, orgânico e convencional, mesmo que preliminar, é muito importante, dada à pequena porcentagem da área de cenoura que é produzida em sistemas agroecológicos, e à dificuldade de seleção para queima-das-folhas em cultivo orgânico devido à pequena pressão de inóculo e a impossibilidade de inoculação de patógenos nessas áreas, possibilitando também a diminuição de recursos financeiros e de mão-de-obra empregados no melhoramento.

\section{REFERÊNCIAS}

CRUZ CD. 1997. Programa Genes: aplicativo computacional em genética e estatística. Viçosa: UFV. 442p.

CRUZ CD; REGAZZI AJ. 2001. Modelos biométricos aplicados ao melhoramento genético. Viçosa: Editora UFV. 390p.

LAMMERTS VAN BUEREN ET; HULSCHER M; HARING M; JONGERDEN J; VAN
MANSVELT JD; DEN NIJS APM; RUIVENKAMP GTP. 1999. Sustainable organic plant breeding. Final report: a vision, choices, consequences and steps. Driebergen: Louis Bolk Institut. 60p.

MICHALIK B; ZABAGALO A; ZUKOWSKA E. 1985. Investigation of the interdependence of root color and carotene content in carrot variety Selecta. Plant Breeding Abstract 55: 312-316.

MINOLTA. 1994. Precise color communication: color control from feeling to instrumentation. Japão: $49 \mathrm{p}$.

ONOYAMA SS; SILVA GO; MOITA AW; VIEIRA JV; LOPES CA; SOUZA GS. 2011. Principais características da raiz de cenoura na perspectiva de agentes da cadeia produtiva. Horticultura Brasileira 29: 387-391.

ORMOND JGP; PAULA SRL; FILHO PF; ROCHA LTM. 2002. Agricultura orgânica: quando o passado é futuro. BNDES Setorial: Rio de Janeiro. 15: 3-34.

PEREIRAAS. 2002. Teores de carotenóides totais em cenoura (Daucus carota L.) e sua relação com a coloração das raizes. Universidade Federal de Viçosa: Viçosa. 128 p. (Tese doutorado).

REIS EF; REIS MS; CRUZ CD; SEDIYAMA T. 2004. Comparação de procedimentos de seleção para produção de grãos em populações de soja. Ciência Rural 34: 685-691.

RESENDE FV; VIEIRA JV; VIDAL MC. 2005. Avaliação de populações de cenoura do programa de melhoramento da Embrapa Hortaliças em sistema orgânico de produção. In: CONGRESSO BRASILEIRO DE OLERICULTURA, 45. Resumos... Fortaleza: SOB (CD-ROM).

SAMINEZ TCO; RESENDE FV; VIEIRA JV; COUTO JR; PAULA WS; LIMA DB. 2002. Desempenho de cultivares e populações de cenoura em cultivo orgânico no verão do Distrito Federal. In: CONGRESSO BRASILEIRO DE OLERICULTURA, 42. Resumos... Uberlândia: SOB (CD-ROM).

SCOTT A J; KNOTT M. 1974. A cluster analysis method for grouping means in the analysis of variance. Biometrics 30: 507-512.

SILVA GO; VIEIRA JV. 2008. Componentes genéticos e fenotípicos para caracteres de importância agronômica em população de cenoura sob seleção recorrente. Horticultura Brasileira 26: 481-485.

SILVA GO; VIEIRA JV. 2009. Ganhos realizados com a seleção para caracteres de importância agronômica em cenoura. Horticultura Brasileira 27: 453-457.

SILVA GO; VIEIRA JV. 2010. Ganhos genéticos após seis ciclos de seleção em três populações de cenoura. Revista Ceres 57: 768-772.

SILVA GO; VIEIRA JV; VILELA MS. 2009. Seleção de caracteres de cenoura cultivada em dois sistemas de produção agroecológicos no Distrito Federal. Revista Ceres 56: 595-601.

SILVA GO; VIEIRA JV; VILLELA MS. 2009b. Tamanho de amostra para avaliação de caracteres de cenoura em sistemas de cultivo agroecológico. Horticultura Brasileira 27: $166-170$ 
VIEIRA JV; SILVA GO da; BOITEUX LS. 2012. Genetic parameter and correlation estimates of processing traits in half-sib progenies of tropical-adapted carrot germplasm. Horticultura Brasileira 30: 7-11.

SIMON PW; PETERSON CE. 1993. Genetic and environmental components of carrot culinary and nutritive value. In: SYMPOSIUM ON QUALITY OF VEGETABLES, 93. Anais... Lund: ISHS Acta Horticulturae. p. 165-167.

SOUZA JL. 2006. Manual de horticultura orgânica. 2.ed. atual e ampl. Viçosa: Aprenda Fácil. 843p.

SOUZA RB; ALCÂNTARAFA. 2008. Adubação no sistema orgânico de produção de hortaliças.
Brasilia-DF: Embrapa Hortaliças, (Circular técnica 65 , Publicação eletrônica).

VIEIRA JV; OLIVEIRA VR. 2003. Interação de genótipos com ambientes em cenoura Brasília. Melhoramento da qualidade de vida. In: CONGRESSO BRASILEIRO DE MELHORAMENTO DE PLANTAS, 2. Resumos... Porto Seguro: SBMP (CD-ROM).

VIEIRA JV; SILVA GO; BOITEUX LS; SIMON P. 2009. Divergência genética entre acessos de cenoura pertencentes a grupos varietais distintos utilizando caracteres morfológicos. Horticultura Brasileira 27: 468-472.

VIEIRA JV; SILVA GO; BOITEUX LS. 2012. Genetic parameter and correlation estimates of processing traits in half-sib progenies of tropical-adapted carrot germplasm. Horticultura Brasileira 30: 7-11.

WHITE JM; STRANDBERG JO. 1978. Early root growth of carrots in organic soil. Journal of American Society of Horticultural Science 103: 344-347.

WOLFE MS. 2003. Plant breeding, ecology and modern organic agriculture. In: PROCEEDINGS OF ECO-PB 1 rst INTERNATIONAL SYMPOSIUM ON ORGANIC SEED PRODUCTION AND PLANT BREEDING, 1. Anais... Berlim: ECO-PB. p. 21-22. 